Cahiers $d u$ MONDE RUSSE

\section{Cahiers du monde russe}

Russie - Empire russe - Union soviétique et États indépendants

$46 / 4 \mid 2005$

L'invention d'une politique humanitaire

\title{
Ernest A. Zitser, The Transfigured Kingdom
}

\section{Aleksandr Lavrov}

\section{OpenEdition \\ Journals}

Édition électronique

URL : https://journals.openedition.org/monderusse/6564

DOI : 10.4000/monderusse. 6564

ISSN : $1777-5388$

\section{Éditeur}

Éditions de l'EHESS

\section{Édition imprimée}

Date de publication : 1 décembre 2005

Pagination : 862-864

ISBN : 2-7132-2057-2

ISSN : $1252-6576$

\section{Référence électronique}

Aleksandr Lavrov, «Ernest A. Zitser, The Transfigured Kingdom », Cahiers du monde russe [En ligne], 46/4 | 2005, mis en ligne le 29 juin 2009, consulté le 03 septembre 2022. URL : http://

journals.openedition.org/monderusse/6564; DOI : https://doi.org/10.4000/monderusse.6564

Ce document a été généré automatiquement le 3 septembre 2022

Tous droits réservés 


\title{
Ernest A. Zitser, The Transfigured Kingdom
}

\author{
Aleksandr Lavrov
}

\section{RÉFÉRENCE}

Ernest A. ZITSER, The Transfigured Kingdom. Sacred Parody and Charismatic Authority at the Court of Peter the Great. Ithaca, Londres : Cornell University Press, 2004, $221 \mathrm{p}$.

1 Enfin une étude du « synode très bouffon » (vsešutejšij sobor) de Pierre le Grand! Le livre de Ernest A. Zitser devrait susciter l'intérêt des spécialistes de la période. Traité au XIX ${ }^{e}$ siècle par Mihail I. Semevskij, ce sujet a connu un regain d'actualité au cours des vingt dernières années grâce aux travaux de Boris A. Uspenskij. L'auteur discerne dans l'historiographie du "synode très bouffon » une interprétation " utilitariste » (qui met l'accent sur l'importance des jeux militaires) et une interprétation « propagandiste » (le synode ferait partie d'une entreprise de sécularisation, et serait dirigé par conséquent contre le clergé).

2 L'auteur les rejette toutes les deux, ainsi que l'explication, proposée par Uspenskij, du synode comme exemple de " conduite d'inversion» (antibehaviour). L'argumentation de Zitser est remarquable: «... La nature binaire des divisions, implicite dans cette formulation (passé/futur, tradition/modernité, russe/occidental), montre à quel point cette interprétation, tributaire des polarités structuralistes, doit plus encore aux débats contemporains sur une voie spécifique de la Russie - débats qui ont moins à voir avec la conception du monde de Pierre le Grand et de ses contemporains qu'avec les constructions idéologiques actuelles » (p. 14).

3 On peut regretter cependant que Zitser n'ait pas pris en considération un autre article, plus positiviste, d'Uspenskij et B. Šiškin, qui souligne les tendances anti-catholiques des mascarades péterbourgeoises ${ }^{1}$. Car il signale parfois lui-même les allusions anticatholiques de certains divertissements (par exemple, le chapeau rouge de cardinal 
romain porté par le fiancé pendant le mariage de Nikita Zotov en 1715, p. 131), mais sans les remettre dans leur contexte.

4 Selon la conception, très originale, de l'auteur, le « synode très bouffon » est avant tout un élément de légitimation et de représentation du tsar-réformateur. Pierre le Grand a inventé un "monde pour rire »- avec des bouffons en guise de rois, de chevaliers et d'ecclésiastiques, avec des cérémonies extravagantes destinées à renforcer la corporation, et avec une topographie imaginaire - qui servait « de point de référence important pour tous les membres du cercle des intimes du tsar». Pour expliquer le fonctionnement de ce monde, l'auteur introduit la notion de "royaume transfiguré » (transfigured kingdom). L'expression est tirée du nom du village où se déroulèrent les premiers jeux militaires du jeune tsar (Preobraženskoe: village de laTransfiguration). « Ce royaume imaginaire séparait tous ceux qui en étaient venus à croire au don de grâce de Pierre » (c'est-à-dire à son charisme, au sens originel, religieux et non weberien) « de ceux qui n'étaient toujours pas convaincus ou demeuraient hostiles [...]. Les sacrements politiques liés au "royaume transfiguré" de Pierre devaient élever la personne du tsar audessus des factions de la cour et de la politique des clans, réaffirmer son droit d'intervention dans les affaires ecclésiastiques et transformer son entourage en une communauté œcuménique de vrais croyants. En impliquant les courtisans dans des mystères bachiques destinés à briser les tabous, le tsar et ses conseillers voulaient imposer un nouveau mode de distinction entre "noble" et "vil", entre sacré et profane, entre tradition et modernité aux membres choisis de l'élite moscovite» (p. 4-5).

5 Cette interprétation suscite un certain nombre de questions. En premier lieu, même s'il existe, dans l'histoire de la culture russe, quantité de messages envoyés dans le vide, celui du " royaume transfiguré » était d'une telle importance que sa réception devient un problème capital. Quelle fut la réaction des représentants de l'élite, qui ne partageaient pas tous, loin de là, la conception des réformes qui était celle de Pierre I ${ }^{\text {er }}$ ? L'auteur n'évoque ici que des exemples connus, comme le pamphlet contre le synode (1705) et le cas du prince Ivan I. Hovanskij (p. 2-3, 74, 170). Mais quelle fut la réaction du "peuple», c'est-à-dire des acteurs de la «culture populaire»? L'auteur ne s'en préoccupe guère, alors pourtant que les documents existent: la chancellerie de Preobraženskoe (Chancellery of Transfiguration, écrit Zitser, p. 61) recueillait minutieusement tous les propos critiques à l'égard du tsar et de son entourage. Même si la délimitation du sujet implique de n'envisager le phénomène des mascarades qu'à travers les yeux du tsar, on peut se demander si la réaction de ses sujets l'intéressait ou non.

6 En second lieu, les tentatives de Zitser pour trouver dans les discussions des théologiens orthodoxes des traces d'une "théologie politique » sont parfois très audacieuses. C'est dans ce sens qu'il interprète le débat des années 1680 sur la Transsubstantiation et celui qui opposa Feofan Prokopovič à Feofilakt Lopatinskij sur la justification - par les œuvres ou par la foi (p. 40, 147-149). Ma position à cet égard est beaucoup plus traditionnelle et réservée. Il me semble que la discussion sur la Transsubstantiation, qui, comme Wolfram von Scheliha a tenté de le montrer récemment, était la première vraie discussion "scolastique » en Moscovie ${ }^{2}$, portait sur plusieurs points - par exemple, sur le choix entre deux orientations, latine ou grecque, des études supérieures, ou sur les méthodes de discussion en théologie - et ne peut pas être réduite au seul problème de la « légitimation ». L'influence de ces débats sur la sphère politique, que je ne veux pas nier, était beaucoup plus indirecte. 
7 L'auteur réussit par ailleurs à établir une base de données prosopographiques qui lui permet de reconstruire avec minutie la liste des participants au " synode très bouffon ". Il introduit dans l'histoire du synode l'épisode oublié de l'Ordre de Judas dont fut décoré l'hetman Ivan Mazepa. Il faut avouer que cette histoire amène de l'eau à son moulin, puisque l'assimilation de Mazepa à Judas impliquerait celle de Pierre le Grand au Sauveur. Très intéressante également est l'analyse des trois mariages - de Filat Šanskij (1702), du tsar (1711) et de Nikita Zotov (1715). Zitser montre comment le «synode très bouffon" aida Pierre le Grand à rééduquer les élites et à remplacer les rituels moscovites par des cérémonies à l'européenne. La remarque selon laquelle le synode "disparaît" avec son créateur (p.173) est cruciale pour la conception de l'auteur. Mais la «Maison de Glace» (Ledjanoj dom, 1740) ne se trouve-t-elle pas également dans le « royaume transfiguré »?

Il faut bien noter quelques erreurs de fait. Fedor Šaklovityj, présenté par l'auteur comme le directeur de la police moscovite (p. 27), n'était que le chef du secrétariat des strel'cy (Streleckij prikaz), et non du secrétariat de la Ville (Zemskij prikaz), qui était responsable des fonctions de police à Moscou au XVII siècle ${ }^{3}$. L'interprétation par Zitser de la lettre du tsar Pierre à son frère Ivan (1689) comme un « manifeste des Naryškiny » (p. 26-35) ne semble pas aller de soi, puisque cette lettre ne fut pas publiée à l'époque et que son contenu ne fut connu que de quelques personnes. L'adhésion des parents de Anna Stremouhova (née Paškova) à la vieille foi ne permet pas de la définir elle aussi comme appartenant aux vieux-croyants (p. 128, 134).

Mais ces menues imprécisions sont sans gravité. Il me semble en revanche que l'auteur s'est fixé comme objectif d'inscrire son œuvre de manière trop stricte dans le contexte de l'historiographie actuelle, notamment dans l'optique des travaux de Richard Wortman, dont il utilise aussi la notion de «scénarios du pouvoir» (p. 5). Dans le même temps, il tranche brusquement les liens qui l'attachent à l'historiographie classique, et surtout à la conception de Bahtin, qui n'est évoqué que dans la bibliographie, alors que la terminologie de Zitser (play world) est parfois bakhtinienne, ainsi que ses remarques à propos du caractère carnavalesque de certains rituels du "synode très bouffon ». Par exemple, à propos du mariage de Zotov, l'auteur remarque que le «mariage mal assorti" est un thème traditionnel $\mathrm{du}$ charivari, "cérémonie traditionnelle d'humiliation, imposée par ceux qui s'étaient proclamés gardiens de la morale à ceux qui violaient l'ordre social, sexuel et confessionnel dominant en Europe au début des Temps modernes » (p. 134-135). Et le mannequin de paille de Mazepa décrit par l'auteur (p. 95-96) n'est-il pas comparable à une Kostroma, bien connue de l'ethnographie des Slaves orientaux?

10 Cependant, même si l'on peut reprocher à Zitser de n'avoir sélectionné pour son étude, de manière du reste délibérée et cohérente, que les phénomènes de la « culture élitaire », il n'en reste pas moins qu'il a le mérite d'avoir conçu un ouvrage dont la lecture est indispensable à quiconque travaille sur la Russie de Pierre le Grand. 


\section{NOTES}

1. B. Šiškin, B. A. Uspenskij, «Tred'jakovskij i žansenisty », Simvol, 23, 1990, p. 105-264.

2. Wolfram von Scheliha, «The Orthodox Universal Church and the Emergence of Intellectual Life in Muscovite Russia ", in Hans-Joachim Torke, ed., Von Moskau nach St. Petersburg. Das russische Reich im 17. Jahrhundert, Wiesbaden, 2000, p. 273-289, ici p. 286-288 (Forschungen zur osteuropäischen Geschichte, Bd. 56).

3. S. K. Bogojavlenskij, Prikaznye sud'i XVII v., M.-L., 1946, p. 167-168. 